\title{
Positive Implicative Ideals of BCK-Algebras Based on Intersectional Soft Sets
}

\author{
Eun Hwan Roh ${ }^{1}$ and Young Bae Jun ${ }^{2}$ \\ ${ }^{1}$ Department of Mathematics Education, Chinju National University of Education, Jinju 660-756, Republic of Korea \\ ${ }^{2}$ Department of Mathematics Education (and RINS), Gyeongsang National University, Jinju 660-701, Republic of Korea
}

Correspondence should be addressed to Eun Hwan Roh; idealmath@gmail.com

Received 14 September 2012; Revised 17 March 2013; Accepted 8 May 2013

Academic Editor: Mina Abd-El-Malek

Copyright ( 2013 E. H. Roh and Y. B. Jun. This is an open access article distributed under the Creative Commons Attribution License, which permits unrestricted use, distribution, and reproduction in any medium, provided the original work is properly cited.

\begin{abstract}
The aim of this paper is to lay a foundation for providing a soft algebraic tool in considering many problems that contain uncertainties. In order to provide these soft algebraic structures, the notion of int-soft positive implicative ideals is introduced, and related properties are investigated. Relations between an int-soft ideal and an int-soft positive implicative ideal are established. Characterizations of an int-soft positive implicative ideal are obtained. Extension property for an int-soft positive implicative ideal is constructed. The $\wedge$-product and $\vee$-product of int-soft positive implicative ideals are considered, and the soft intersection (resp., union) of int-soft positive implicative ideals is discussed.
\end{abstract}

\section{Introduction}

Various problems in many fields involve data containing uncertainties which dealt with wide range of existing theories such as the theory of probability, (intuitionistic) fuzzy set theory, vague sets, theory of interval mathematics, and rough set theory. All of these theories have their own difficulties which are pointed out in [1]. To overcome these difficulties, Molodtsov [1] introduced the soft set theory as a new mathematical tool for dealing with uncertainties that is free from the difficulties. Molodtsov successfully applied the soft set theory in several directions, such as smoothness of functions, game theory, operations research, Riemann integration, Perron integration, probability, and theory of measurement (see [1-4]). Soft set theory is applied to algebraic structures, and many algebraic properties of soft sets are studied (see [5-16]). Jun et al. introduced the notion of int-soft sets and applied the notion of soft set theory to BCK/BCI-algebras (see $[14,17,18])$. In the paper [17], the notion of int-soft BCK/BCIalgebras is discussed, and in the paper [18], the notion of int-soft ideals of BCK/BCI-algebras is introduced, and a few results are considered.

As a continuation of the papers $[17,18]$, in this paper, we first investigate more properties of int-soft ideals in BCKalgebras. We introduce the new notion, the so-called int-soft positive implicative ideals in BCK-algebras, and investigate related properties. We consider relations between an int-soft ideal and an int-soft positive implicative ideal and establish characterizations of an int-soft positive implicative ideal. We construct extension property for an int-soft positive implicative ideal. We deal with the $\wedge$-product and $\vee$-product of int-soft positive implicative ideals and discuss the soft intersection (resp., union) of int-soft positive implicative ideals.

\section{Preliminaries}

A BCK/BCI-algebra is an important class of logical algebras introduced by $\mathrm{K}$. Iséki and was extensively investigated by several researchers.

An algebra $(X ; *, 0)$ of type $(2,0)$ is called a $B C I$-algebra if it satisfies the following conditions:

(I) (for all $x, y$, and $z \in X)(((x * y) *(x * z)) *(z * y)=0)$,

(II) (for all $x, y \in X)((x *(x * y)) * y=0)$,

(III) (for all $x \in X)(x * x=0)$,

(IV) (for all $x, y \in X)(x * y=0, y * x=0 \Rightarrow x=y$ ). 
If a BCI-algebra $X$ satisfies the following identity:

(V) (for all $x \in X)(0 * x=0)$,

then $X$ is called a $B C K$-algebra. Any BCK/BCI-algebra $X$ satisfies the following axioms:

(a1) (for all $x \in X)(x * 0=x)$,

(a2) (for all $x, y$, and $z \in X)(x \leq y \Rightarrow x * z \leq y * z, z *$ $y \leq z * x)$,

(a3) (for all $x, y$, and $z \in X)((x * y) * z=(x * z) * y)$,

(a4) (for all $x, y$, and $z \in X)((x * z) *(y * z) \leq x * y)$,

where $x \leq y$ if and only if $x * y=0$.

A nonempty subset $S$ of a BCK/BCI-algebra $X$ is called a subalgebra of $X$ if $x * y \in S$ for all $x, y \in S$. A subset $I$ of a BCK/BCI-algebra $X$ is called an ideal of $X$ if it satisfies

$$
0 \in I
$$

$$
(\forall x \in X)(\forall y \in I) \quad(x * y \in I \Longrightarrow x \in I) \text {. }
$$

A subset $I$ of a BCK-algebra $X$ is called a positive implicative ideal (briefly, PI-ideal) of $X$ if it satisfies (1) and

$$
(\forall x, y, z \in X) \quad((x * y) * z \in I, y * z \in I \Longrightarrow x * z \in I) .
$$

Note that every PI-ideal is an ideal, but the converse is not true (see [19]).

We refer the reader to the books $[19,20]$ for further information regarding $\mathrm{BCK} / \mathrm{BCI}$-algebras.

Molodtsov [1] defined the soft set in the following way. Let $U$ be an initial universe set and $E$ a set of parameters. Let $\mathscr{P}(U)$ denote the power set of $U$ and $A, B, C, \ldots \subseteq E$.

A pair $(\alpha, A)$ is called a soft set over $U$, where $\alpha$ is a mapping given by

$$
\alpha: A \longrightarrow \mathscr{P}(U)
$$

In other words, a soft set over $U$ is a parameterized family of subsets of the universe $U$. For $\varepsilon \in A, \alpha(\varepsilon)$ may be considered as the set of $\varepsilon$-approximate elements of the soft set $(\alpha, A)$. Clearly, a soft set is not a set. For illustration, Molodtsov considered several examples in [1]. We refer the reader to the papers $[1,21-23]$ for further information regarding soft sets.

Let $(\alpha, A)$ and $(\alpha, B)$ be soft sets over $U$. The $\wedge$-product of $(\alpha, A)$ and $(\alpha, B)$ is defined to be a soft set $\left(\alpha_{A \wedge B}, A \times B\right)$ over $U$ which is defined by

$$
\alpha_{A \wedge B}: A \times B \longrightarrow \mathscr{P}(U), \quad(x, y) \longmapsto \alpha(x) \cap \alpha(y) .
$$

The $\vee$-product of $(\alpha, A)$ and $(\alpha, B)$ is defined to be a soft set $\left(\alpha_{A \vee B}, A \times B\right)$ over $U$ which is defined by

$$
\alpha_{A \vee B}: A \times B \longrightarrow \mathscr{P}(U), \quad(x, y) \longmapsto \alpha(x) \cup \alpha(y) .
$$

Assume that $E$ has a binary operation $\hookrightarrow$. For any nonempty subset $A$ of $E$, a soft set $(\alpha, A)$ over $U$ is called an int-soft set over $U$ (see $[17,18])$ if it satisfies

$$
(\forall x, y \in A) \quad(x \hookrightarrow y \in A \Longrightarrow \alpha(x) \cap \alpha(y) \subseteq \alpha(x \hookrightarrow y))
$$

For a soft set $(\alpha, A)$ over $U$ and a subset $\gamma$ of $U$, the $\gamma$ inclusive set of $(\alpha, A)$, denoted by $i(\alpha ; \gamma)$, is defined to be the set

$$
i(\alpha ; \gamma):=\{x \in A \mid \gamma \subseteq \alpha(x)\}
$$

\section{Intersectional Soft Positive Implicative Ideals}

In what follows, we take $E=X$, as a set of parameters, which is a BCK-algebra unless otherwise specified.

Definition 1 (see [18]). A soft set $(\alpha, X)$ over $U$ is called an int-soft algebra over $U$ if it satisfies

$$
(\forall x, y \in X) \quad(\alpha(x * y) \supseteq \alpha(x) \cap \alpha(y)) .
$$

Definition 2 (see [24]). A soft set $(\alpha, X)$ over $U$ is called an int-soft ideal over $U$ if it satisfies

$$
\begin{gathered}
(\forall x \in X) \quad(\alpha(0) \supseteq \alpha(x)), \\
(\forall x, y \in X) \quad(\alpha(x) \supseteq \alpha(x * y) \cap \alpha(y)) .
\end{gathered}
$$

Let $(\alpha, X)$ be a soft set over $U$ and $w$ a fixed element of $X$. If $(\alpha, X)$ is an int-soft ideal over $U$, then $0 \in i(\alpha ; \alpha(w))$ by (10). We have the following question.

Question. Let $(\alpha, X)$ be a soft set over $U$. If $(\alpha, X)$ satisfies the condition (10), then is the $\alpha(w)$-inclusive set $i(\alpha ; \alpha(w))$ an ideal of $X$ ?

The following example provides a negative answer to this question; that is, there exists an element $u \in X$ such that $i(\alpha ; \alpha(u))$ is not an ideal of $X$.

Example 3. Let $X=\{0, a, b, c, d\}$ be a BCK-algebra with the following Cayley table:

\begin{tabular}{l|lllll}
$*$ & 0 & $a$ & $b$ & $c$ & $d$ \\
\hline 0 & 0 & 0 & 0 & 0 & 0 \\
$a$ & $a$ & 0 & $a$ & 0 & 0 \\
$b$ & $b$ & $b$ & 0 & $b$ & 0 \\
$c$ & $c$ & $c$ & $c$ & 0 & $c$ \\
$d$ & $d$ & $d$ & $d$ & $d$ & 0
\end{tabular}


Let $\gamma_{1}, \gamma_{2}, \gamma_{3}, \gamma_{4}$, and $\gamma_{5}$ be subsets of $U$ such that $\gamma_{1} \supsetneq \gamma_{2} \supsetneq \gamma_{3} \supsetneq$ $\gamma_{4} \supsetneq \gamma_{5}$. Define a soft set $(\alpha, X)$ over $U$ by

$$
\alpha: X \longrightarrow \mathscr{P}(U), \quad x \longmapsto \begin{cases}\gamma_{1} & \text { if } x=0, \\ \gamma_{2} & \text { if } x=a, \\ \gamma_{4} & \text { if } x=b, \\ \gamma_{5} & \text { if } x=c, \\ \gamma_{3} & \text { if } x=d .\end{cases}
$$

Then $(\alpha, X)$ satisfies the condition (10), but it is not an int-soft ideal over $U$ since

$$
\alpha(b)=\gamma_{4} \nsupseteq \gamma_{3}=\alpha(b * d) \cap \alpha(d),
$$

and $i(\alpha ; \alpha(d))=\{0, a, d\}$ is not an ideal of $X$. Note that $i(\alpha ; \alpha(b))=\{0, a, b, d\}$ is an ideal of $X$.

We give conditions for the $\alpha(w)$-inclusive set to be an ideal.

Theorem 4. Let $(\alpha, X)$ be a soft set over $U$ and $w$ a fixed element of $X$. If $(\alpha, X)$ is an int-soft ideal over $U$, then the $\alpha(w)$ inclusive set $i(\alpha ; \alpha(w))$ is an ideal of $X$.

Proof. Recall that $0 \in i(\alpha ; \alpha(w))$. Let $x, y \in X$ be such that $x * y \in i(\alpha ; \alpha(w))$ and $y \in i(\alpha ; \alpha(w))$. Then $\alpha(w) \subseteq \alpha(x * y)$ and $\alpha(w) \subseteq \alpha(y)$. Since $(\alpha, X)$ is an int-soft ideal over $U$, it follows from (11) that

$$
\alpha(x) \supseteq \alpha(x * y) \cap \alpha(y) \supseteq \alpha(w),
$$

which shows that $x \in i(\alpha ; \alpha(w))$. Therefore the $\alpha(w)$-inclusive set $i(\alpha ; \alpha(w))$ is an ideal of $X$.

Theorem 5. Let $(\alpha, X)$ be a soft set over $U$ and $w \in X$. Then the following hold.

(1) If the $\alpha(w)$-inclusive set $i(\alpha ; \alpha(w))$ is an ideal of $X$, then $(\alpha, X)$ satisfies the following condition:

$$
\begin{gathered}
(\forall x, y, z \in X) \\
(\alpha(x) \subseteq \alpha(y * z) \cap \alpha(z) \Longrightarrow \alpha(x) \subseteq \alpha(y)) .
\end{gathered}
$$

(2) If $(\alpha, X)$ satisfies (10) and (16), then the $\alpha(w)$-inclusive set $i(\alpha ; \alpha(w))$ is an ideal of $X$.

Proof. (1) Assume that the $\alpha(w)$-inclusive set $i(\alpha ; \alpha(w))$ is an ideal of $X$. Let $x, y$, and $z \in X$ be such that $\alpha(x) \subseteq \alpha(y * z) \cap$ $\alpha(z)$. Then $y * z \in i(\alpha ; \alpha(x))$ and $z \in i(\alpha ; \alpha(x))$. It follows from (2) that $y \in i(\alpha ; \alpha(x))$; that is, $\alpha(x) \subseteq \alpha(y)$. Hence (16) is valid.

(2) Suppose that $(\alpha, X)$ satisfies (10) and (16). Obviously $0 \in i(\alpha ; \alpha(w))$ by (10). Let $x, y \in X$ be such that $x * y \in$ $i(\alpha ; \alpha(w))$ and $y \in i(\alpha ; \alpha(w))$. Then $\alpha(x * y) \supseteq \alpha(w)$ and $\alpha(y) \supseteq \alpha(w)$, which imply that $\alpha(w) \subseteq \alpha(x * y) \cap \alpha(y)$. It follows from (16) that $\alpha(w) \subseteq \alpha(x)$; that is, $x \in i(\alpha ; \alpha(w))$. Therefore $i(\alpha ; \alpha(w))$ is an ideal of $X$.
Definition 6. A soft set $(\alpha, X)$ over $U$ is called an int-soft PIideal over $U$ if it satisfies (10) and

$$
(\forall x, y, z \in X) \quad(\alpha(x * z) \supseteq \alpha((x * y) * z) \cap \alpha(y * z)) .
$$

Example 7. Let $X=\{0, a, b\}$ be a BCK-algebra with the following Cayley table:

$$
\begin{array}{l|lll}
* & 0 & a & b \\
\hline 0 & 0 & 0 & 0 \\
a & a & 0 & 0 \\
b & b & b & 0
\end{array}
$$

Let $\gamma_{1}, \gamma_{2}$, and $\gamma_{3}$ be subsets of $U$ such that $\gamma_{1} \supsetneq \gamma_{2} \supsetneq \gamma_{3}$. Define a soft set $(\alpha, X)$ over $U$ by

$$
\alpha: X \longrightarrow \mathscr{P}(U), \quad x \longmapsto \begin{cases}\gamma_{1} & \text { if } x=0 \\ \gamma_{2} & \text { if } x=a, \\ \gamma_{3} & \text { if } x=b .\end{cases}
$$

Then $(\alpha, X)$ is an int-soft PI-ideal over $U$.

Example 8. Let $X=\{0,1,2,3,4\}$ be a BCK-algebra with the following Cayley table:

\begin{tabular}{l|lllll}
$*$ & 0 & 1 & 2 & 3 & 4 \\
\hline 0 & 0 & 0 & 0 & 0 & 0 \\
1 & 1 & 0 & 0 & 1 & 0 \\
2 & 2 & 2 & 0 & 2 & 0 \\
3 & 3 & 3 & 3 & 0 & 3 \\
4 & 4 & 4 & 4 & 4 & 0
\end{tabular}

Let $\left\{\gamma_{1}, \gamma_{2}, \gamma_{3}, \gamma_{4}, \gamma_{5}\right\}$ be a class of subsets of $U$ which is a poset with the following Hasse diagram (see Figure 1).

Let $\alpha$ be a soft set over $U$ defined by

$$
\alpha: E \longrightarrow \mathscr{P}(U), \quad x \longmapsto \begin{cases}\gamma_{5} & \text { if } x=0, \\ \gamma_{4} & \text { if } x=1, \\ \gamma_{3} & \text { if } x=2, \\ \gamma_{2} & \text { if } x=3, \\ \gamma_{1} & \text { if } x=4 .\end{cases}
$$

Then $(\alpha, X)$ is an int-soft PI-ideal over $U$.

We discuss relations between int-soft ideal and int-soft PI-ideal.

Theorem 9. Every int-soft PI-ideal is an int-soft ideal.

Proof. Let $(\alpha, X)$ be an int-soft PI-ideal over $U$. If we take $z=$ 0 in (17) and use (a1), then we have (11). Hence $(\alpha, X)$ is an int-soft ideal over $U$.

The converse of Theorem 9 is not true as seen in the following example. 


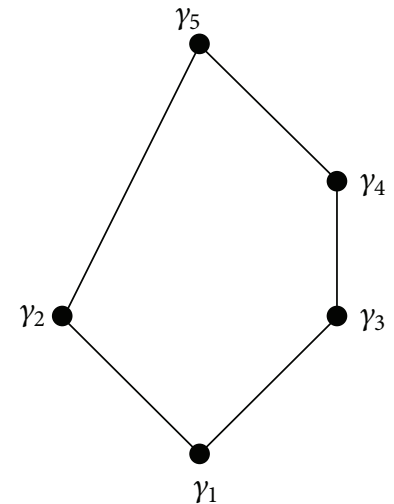

FIGURE 1

Example 10. Let $X=\{0, a, b, c\}$ be a BCK-algebra with the following Cayley table:

$$
\begin{array}{l|llll}
* & 0 & a & b & c \\
\hline 0 & 0 & 0 & 0 & 0 \\
a & a & 0 & 0 & a \\
b & b & a & 0 & b \\
c & c & c & c & 0
\end{array}
$$

Let $\gamma_{1}, \gamma_{2}$, and $\gamma_{3}$ be subsets of $U$ such that $\gamma_{1} \supsetneq \gamma_{2} \supsetneq \gamma_{3}$. Define a soft set $(\alpha, X)$ over $U$ by

$$
\alpha: X \longrightarrow \mathscr{P}(U), \quad x \longmapsto \begin{cases}\gamma_{1} & \text { if } x=0, \\ \gamma_{2} & \text { if } x \in\{a, b\} \\ \gamma_{3} & \text { if } x=c .\end{cases}
$$

Then $(\alpha, X)$ is an int-soft ideal over $U$. But it is not an int-soft PI-ideal over $U$ since

$$
\alpha(b * a)=\gamma_{2} \nsupseteq \gamma_{1}=\alpha((b * a) * a) \cap \alpha(a * a) .
$$

We provide conditions for an int-soft ideal to be an intsoft PI-ideal.

Lemma 11 (see [18]). Every int-soft ideal ( $\alpha, X)$ over U satisfies

$$
(\forall x, y \in X) \quad(x \leq y \Longrightarrow \alpha(x) \supseteq \alpha(y)) .
$$

Theorem 12. For a soft set $(\alpha, X)$ over $U$, the following are equivalent.

(1) $(\alpha, X)$ is an int-soft PI-ideal over $U$.

(2) $(\alpha, X)$ is an int-soft ideal over $U$ satisfying the condition

$$
(\forall x, y \in X) \quad(\alpha(x * y) \supseteq \alpha((x * y) * y)) .
$$

(3) $(\alpha, X)$ is an int-soft ideal over $U$ satisfying the condition

$(\forall x, y, z \in X) \quad(\alpha((x * z) *(y * z)) \supseteq \alpha((x * y) * z))$.
Proof. Assume that $(\alpha, X)$ is an int-soft PI-ideal over $U$. Then $(\alpha, X)$ is an int-soft ideal over $U$ by Theorem 9 . If we take $z=$ $y$ in (17), then

$$
\begin{aligned}
\alpha(x * y) & \supseteq \alpha((x * y) * y) \cap \alpha(y * y) \\
& =\alpha((x * y) * y) \cap \alpha(0)=\alpha((x * y) * y) .
\end{aligned}
$$

Hence (2) is valid. Now, let $(\alpha, X)$ be an int-soft ideal over $U$ satisfying (26). Note that

$$
\begin{aligned}
& ((x *(y * z)) * z) * z \\
& \quad=((x * z) *(y * z)) * z \leq(x * y) * z
\end{aligned}
$$

for all $x, y$, and $z \in X$. Using Lemma 11, we have

$$
\alpha(((x *(y * z)) * z) * z) \supseteq \alpha((x * y) * z) .
$$

It follows from (a3) and (26) that

$$
\begin{aligned}
\alpha((x * z) *(y * z)) & =\alpha((x *(y * z)) * z) \\
& \supseteq \alpha(((x *(y * z)) * z) * z) \\
& \supseteq \alpha((x * y) * z) .
\end{aligned}
$$

Therefore (3) holds. Finally, let $(\alpha, X)$ be an int-soft ideal over $U$ satisfying (27). Then

$$
\begin{gathered}
\alpha(x * z) \supseteq \alpha((x * z) *(y * z)) \cap \alpha(y * z) \\
\supseteq \alpha((x * y) * z) \cap \alpha(y * z)
\end{gathered}
$$

for all $x, y$, and $z \in X$ by using (11) and (27). Therefore $(\alpha, X)$ is an int-soft PI-ideal over $U$.

Theorem 13. A soft set $(\alpha, X)$ over $U$ is an int-soft PI-ideal over $U$ if and only if $(\alpha, X)$ satisfies (10) and

$$
(\forall x, y, z \in X) \quad(\alpha(x * y) \supseteq \alpha(((x * y) * y) * z) \cap \alpha(z)) .
$$

Proof. Let $(\alpha, X)$ be an int-soft PI-ideal over $U$. Then $(\alpha, X)$ is an int-soft ideal over $U$ by Theorem 9 . Hence the condition (10) holds. Using (11), (a1), (a3), (III), and (27), we have

$$
\begin{aligned}
\alpha(x * y) & \supseteq \alpha((x * y) * z) \cap \alpha(z) \\
& =\alpha(((x * z) * y) *(y * y)) \cap \alpha(z) \\
& \supseteq \alpha(((x * z) * y) * y) \cap \alpha(z) \\
& =\alpha(((x * y) * y) * z) \cap \alpha(z)
\end{aligned}
$$

for all $x, y$, and $z \in X$, which proves (33).

Conversely, assume that a soft set $(\alpha, X)$ over $U$ satisfies two conditions (10) and (33). Then

$$
\begin{aligned}
\alpha(x)=\alpha(x * 0) & \supseteq \alpha(((x * 0) * 0) * z) \cap \alpha(z) \\
& =\alpha(x * z) \cap \alpha(z)
\end{aligned}
$$


for all $x, z \in X$. Hence $(\alpha, X)$ is an int-soft ideal over $U$. If we take $z=0$ in (33), then

$$
\begin{aligned}
\alpha(x * y) & \supseteq \alpha(((x * y) * y) * 0) \cap \alpha(0) \\
& =\alpha((x * y) * y) \cap \alpha(0)=\alpha((x * y) * y)
\end{aligned}
$$

for all $x, y \in X$ by (al) and (10). It follows from Theorem 12 that $(\alpha, X)$ is an int-soft PI-ideal over $U$.

Lemma 14. A soft set $(\alpha, X)$ over $U$ is an int-soft ideal over $U$ if and only if it satisfies

$$
(\forall x, y, z \in X) \quad(x * y \leq z \Longrightarrow \alpha(x) \supseteq \alpha(y) \cap \alpha(z)) .
$$

Proof. Necessity follows from [18, Proposition 3.7]. Assume that $(\alpha, X)$ satisfies (37). Since $0 * x \leq x$ for all $x \in X$, we have $\alpha(0) \supseteq \alpha(x)$ for all $x \in X$. Note that $(x *(x * y)) * y=0$, that is, $x *(x * y) \leq y$, for all $x, y \in X$. It follows from (37) that $\alpha(x) \supseteq \alpha(x * y) \cap \alpha(y)$ for all $x, y \in X$. Hence $(\alpha, X)$ is an int-soft ideal over $U$.

The following could be easily proved by induction.

Corollary 15. A soft set $(\alpha, X)$ over $U$ is an int-soft ideal over $U$ if and only if it satisfies, for all $x, a_{1}, a_{2}, \ldots, a_{n} \in X$,

$$
x * \prod_{i=1}^{n} a_{i}=0 \Longrightarrow \alpha(x) \supseteq \bigcap_{i=1,2, \ldots, n} \alpha\left(a_{i}\right),
$$

where $x * \prod_{i=1}^{n} a_{i}=\left(\cdots\left(x * a_{1}\right) * \cdots\right) * a_{n}$.

Theorem 16. A soft set $(\alpha, X)$ over $U$ is an int-soft PI-ideal over $U$ if and only if it satisfies

$$
\begin{aligned}
(\forall x, y, a, b \in X) \quad((((x * y) * y) * a) * b \\
\quad=0 \Longrightarrow \alpha(x * y) \supseteq \alpha(a) \cap \alpha(b)) .
\end{aligned}
$$

Proof. Suppose $(\alpha, X)$ is an int-soft PI-ideal over $U$. Then $(\alpha, X)$ is an int-soft ideal over $U$ by Theorem 9. Let $x, y, a$, and $b \in X$ be such that $(((x * y) * y) * a) * b=0$. It follows from (26) and Lemma 14 that $\alpha(x * y) \supseteq \alpha((x * y) *$ $y) \supseteq \alpha(a) \cap \alpha(b)$.

Conversely, assume that $(\alpha, X)$ satisfies the condition (39). For any $x, a$, and $b \in X$, let $(x * a) * b=0$, which is equivalent to $(((x * 0) * 0) * a) * b=0$. Thus

$$
\alpha(x)=\alpha(x * 0) \supseteq \alpha(a) \cap \alpha(b)
$$

by (al) and (39). It follows from Lemma 14 that $(\alpha, X)$ is an int-soft ideal over $U$. Since $(((x * y) * y) *((x * y) * y)) * 0=0$ for all $x, y \in X$, we have

$$
\alpha(x * y) \supseteq \alpha((x * y) * y) \cap \alpha(0)=\alpha((x * y) * y)
$$

by (39) and (10). Therefore $(\alpha, X)$ is an int-soft PI-ideal over $U$ by Theorem 12 .
Theorem 17. A soft set $(\alpha, X)$ over $U$ is an int-soft PI-ideal over $U$ if and only if it satisfies, for all $x, y, z, a$, and $b \in X$,

$$
\begin{aligned}
& (((x * y) * z) * a) * b=0 \\
& \quad \Longrightarrow \alpha((x * z) *(y * z)) \supseteq \alpha(a) \cap \alpha(b) .
\end{aligned}
$$

Proof. Suppose $(\alpha, X)$ is an int-soft PI-ideal over $U$. Then $(\alpha, X)$ is an int-soft ideal over $U$ by Theorem 9. Let $x, y$, $z, a$, and $b \in X$ be such that $(((x * y) * z) * a) * b=0$. It follows from (27) and Lemma 14 that

$$
\alpha((x * z) *(y * z)) \supseteq \alpha((x * y) * z) \supseteq \alpha(a) \cap \alpha(b)
$$

which proves (42).

Conversely, assume that $(\alpha, X)$ satisfies the condition (42). Let $(((x * y) * y) * a) * b=0$ for all $x, y, a$, and $b \in X$. Then

$$
\alpha(x * y)=\alpha((x * y) *(y * y)) \supseteq \alpha(a) \cap \alpha(b)
$$

by (III), (a1), and (42). It follows from Theorem 16 that $(\alpha, X)$ is an int-soft PI-ideal over $U$.

The above two theorems have more general forms.

Theorem 18. A soft set $(\alpha, X)$ over U is an int-soft PI-ideal over $U$ if and only if it satisfies, for all $x, y, a_{1}, a_{2}, \ldots, a_{n} \in X$,

$$
((x * y) * y) * \prod_{i=1}^{n} a_{i}=0 \Longrightarrow \alpha(x * y) \supseteq \bigcap_{i=1,2, \ldots, n} \alpha\left(a_{i}\right) .
$$

Proof. Suppose $(\alpha, X)$ is an int-soft PI-ideal over $U$. Then $(\alpha, X)$ is an int-soft ideal over $U$. Let $x, y, a_{1}, a_{2}, \ldots, a_{n} \in X$ be such that $((x * y) * y) * \prod_{i=1}^{n} a_{i}=0$. By (26) and Corollary 15, we have

$$
\alpha(x * y) \supseteq \alpha((x * y) * y) \supseteq \bigcap_{i=1,2, \ldots, n} \alpha\left(a_{i}\right),
$$

which proves (45).

Conversely, assume that $(\alpha, X)$ satisfies the condition (45). Let $x, y, a$, and $b \in X$ be such that $(((x * y) * y) * a) * b=$ 0 . Then $\alpha(x * y) \supseteq \alpha(a) \cap \alpha(b)$ by (45). It follows from Theorem 16 that $(\alpha, X)$ is an int-soft PI-ideal over $U$.

Theorem 19. A soft set $(\alpha, X)$ over $U$ is an int-soft PI-ideal over $U$ if and only if it satisfies, for all $x, y, z, a_{1}, a_{2}, \ldots, a_{n} \in X$,

$$
\begin{aligned}
& ((x * y) * z) * \prod_{i=1}^{n} a_{i}=0 \\
& \Longrightarrow \alpha((x * z) *(y * z)) \supseteq \bigcap_{i=1,2, \ldots, n} \alpha\left(a_{i}\right) .
\end{aligned}
$$

Proof. It is similar to Theorem 18. 
Lemma 20 (see [18]). Every int-soft ideal is an int-soft algebra.

Theorem 21. A soft set $(\alpha, X)$ over $U$ is an int-soft PI-ideal over $U$ if and only if it satisfies

$$
(\forall \gamma \subseteq U) \quad(i(\alpha ; \gamma) \neq \emptyset \Longrightarrow i(\alpha ; \gamma) \text { is a PI-ideal of } X) .
$$

Proof. Assume that $(\alpha, X)$ is an int-soft PI-ideal over $U$. Then $(\alpha, X)$ is an int-soft ideal over $U$ by Theorem 9 , and so it is an int-soft algebra by Lemma 20. Let $\gamma \subseteq U$ be such that $i(\alpha ; \gamma) \neq \emptyset$. Then there exists $x \in X$ such that $\gamma \subseteq \alpha(x)$. Using (9) and (III), we have

$$
\gamma \subseteq \alpha(x) \cap \alpha(x) \subseteq \alpha(x * x)=\alpha(0),
$$

and so $0 \in i(\alpha ; \gamma)$. Let $x, y$, and $z \in X$ be such that $(x * y) * z \in$ $i(\alpha ; \gamma)$ and $y * z \in i(\alpha ; \gamma)$. Then $\gamma \subseteq \alpha((x * y) * z)$ and $\gamma \subseteq \alpha(y * z)$. It follows from (17) that

$$
\alpha(x * z) \supseteq \alpha((x * y) * z) \cap \alpha(y * z) \supseteq \gamma .
$$

Hence $x * z \in i(\alpha ; \gamma)$. Therefore $i(\alpha ; \gamma)$ is a PI-ideal of $X$.

Conversely, suppose that $i(\alpha ; \gamma)$ is a PI-ideal of $X$ for all $\gamma \subseteq U$ with $i(\alpha ; \gamma) \neq \emptyset$. Then $i(\alpha ; \gamma)$ is a subalgebra of $X$. Let $x, y \in X$ be such that $\alpha(x)=\gamma_{1}$ and $\alpha(y)=\gamma_{2}$. If we take $\gamma=\gamma_{1} \cap \gamma_{2}$, then $x, y \in i(\alpha ; \gamma)$, and so $x * y \in i(\alpha ; \gamma)$. Hence

$$
\alpha(x * y) \supseteq \gamma=\gamma_{1} \cap \gamma_{2}=\alpha(x) \cap \alpha(y) .
$$

If we put $x=y$ in (51) and use (III), then $\alpha(0) \supseteq \alpha(x)$ for all $x \in X$. Let $x, y$, and $z \in X$ be such that $\alpha((x * y) * z)=\gamma_{1}$ and $\alpha(y * z)=\gamma_{2}$. Taking $\gamma=\gamma_{1} \cap \gamma_{2}$ implies that $(x * y) * z \epsilon$ $i(\alpha ; \gamma)$ and $y * z \in i(\alpha ; \gamma)$. It follows from (3) that $x * z \in i(\alpha ; \gamma)$. Thus

$$
\alpha(x * z) \supseteq \gamma=\gamma_{1} \cap \gamma_{2}=\alpha((x * y) * z) \cap \alpha(y * z) .
$$

Therefore $(\alpha, X)$ is an int-soft PI-ideal over $U$.

The PI-ideals $i(\alpha ; \gamma)$ in Theorem 21 are called inclusive PIideals of $X$.

Corollary 22. If $(\alpha, X)$ is an int-soft PI-ideal over $U$, then $i(\alpha ; \gamma)$ is a PI-ideal of $X$ for all $\gamma \subsetneq U$ with $\gamma \subseteq \alpha(0)$.

Theorem 23. For any soft set $(\alpha, X)$ over $U$, let $\left(\alpha^{*}, X\right)$ be a soft set over $U$ defined by

$$
\alpha^{*}: X \longrightarrow \mathscr{P}(U), \quad x \longmapsto \begin{cases}\alpha(x) & \text { if } x \in i(\alpha ; \gamma), \\ \delta & \text { otherwise, }\end{cases}
$$

where $\gamma$ and $\delta$ are subsets of $U$ with $\delta \subsetneq \alpha(x)$. If $(\alpha, X)$ is an int-soft PI-ideal over $U$, then so is $\left(\alpha^{*}, X\right)$.

Proof. Assume that $(\alpha, X)$ is an int-soft PI-ideal over $U$. Then $i(\alpha ; \gamma)$ is a PI-ideal of $X$ for all $\gamma \subseteq U$ with $i(\alpha ; \gamma) \neq \emptyset$. Hence $0 \in i(\alpha ; \gamma)$, and so $\alpha^{*}(0)=\alpha(0) \supseteq \alpha(x) \supseteq \alpha^{*}(x)$ for all $x \in X$. Let $x, y$, and $z \in X$. If $(x * y) * z \in i(\alpha ; \gamma)$ and $y * z \in i(\alpha ; \gamma)$, then $x * z \in i(\alpha ; \gamma)$ by (3). Thus

$$
\begin{aligned}
\alpha^{*}(x * z) & =\alpha(x * z) \supseteq \alpha((x * y) * z) \cap \alpha(y * z) \\
& =\alpha^{*}((x * y) * z) \cap \alpha^{*}(y * z) .
\end{aligned}
$$

If $(x * y) * z \notin i(\alpha ; \gamma)$ or $y * z \notin i(\alpha ; \gamma)$, then $\alpha^{*}((x * y) * z)=\delta$ or $\alpha^{*}(y * z)=\delta$. Hence

$$
\alpha^{*}(x * z) \supseteq \delta=\alpha^{*}((x * y) * z) \cap \alpha^{*}(y * z) .
$$

Therefore $\left(\alpha^{*}, X\right)$ is an int-soft PI-ideal over $U$.

Theorem 24. Every PI-ideal of $X$ can be realized as an inclusive PI-ideal of an int-soft PI-ideals over $U$.

Proof. Let $I$ be a PI-ideal of $X$. Define a soft set $(\alpha, X)$ over $U$ as follows:

$$
\alpha: X \longrightarrow \mathscr{P}(U), \quad x \longmapsto \begin{cases}\gamma & \text { if } x \in I, \\ \emptyset & \text { otherwise }\end{cases}
$$

where $\gamma$ is a nonempty subset of $U$. Clearly, $\alpha(0) \supseteq \alpha(x)$ for all $x \in X$. For every $x, y$, and $z \in X$, if $(x * y) * z \in I$ and $y * z \in I$, then $x * z \in I$. Thus

$$
\alpha(x * z)=\gamma=\alpha((x * y) * z) \cap \alpha(y * z) .
$$

If $(x * y) * z \notin I$ or $y * z \notin I$, then $\alpha((x * y) * z)=\emptyset$ or $\alpha(y * z)=\emptyset$. It follows that

$$
\alpha(x * z) \supseteq \emptyset=\alpha((x * y) * z) \cap \alpha(y * z) .
$$

Therefore $(\alpha, X)$ is an int-soft PI-ideal over $U$, and obviously $i(\alpha ; \gamma)=I$. This completes the proof.

Note that an int-soft ideal might not be an int-soft PIideal (see Example 10). But we have the following extension property for int-soft PI-ideals.

Theorem 25 (extension property for int-soft PI-ideals). Let $(\alpha, X)$ and $(\beta, X)$ be int-soft ideals over $U$ such that $\alpha(0)=$ $\beta(0)$ and $\alpha(x) \subseteq \beta(x)$ for all $x \in X$. If $(\alpha, X)$ is an int-soft $P I$-ideal over $U$, then so is $(\beta, X)$.

Proof. Assume that $(\alpha, X)$ is an int-soft PI-ideal over $U$. For any $x, y$, and $z \in X$, we have

$$
\begin{aligned}
& \beta(((x * z) *(y * z)) *((x * y) * z)) \\
& \quad=\beta(((x * z) *((x * y) * z)) *(y * z)) \\
& \quad=\beta(((x *((x * y) * z)) * z) *(y * z)) \\
& \quad \supseteq \alpha(((x *((x * y) * z)) * z) *(y * z)) \\
& \quad \supseteq \alpha(((x *((x * y) * z)) * y) * z) \\
& \quad=\alpha(((x * y) *((x * y) * z)) * z) \\
& \quad=\alpha(((x * y) * z) *((x * y) * z))=\alpha(0)=\beta(0)
\end{aligned}
$$

by (a3), (27), and (III). It follows from (10) and (11) that

$$
\begin{aligned}
& \beta((x * z) *(y * z)) \\
& \quad=\beta(((x * z) *(y * z)) *((x * y) * z)) \cap \beta((x * y) * z) \\
& \quad \supseteq \beta(0) \cap \beta((x * y) * z)=\beta((x * y) * z)
\end{aligned}
$$


for all $x, y$, and $z \in X$. Therefore, by Theorem $12,(\beta, X)$ is an int-soft PI-ideal over $U$.

Theorem 26. Let $X$ and $Y$ be BCK-algebras. If $(\alpha, X)$ and $(\alpha, Y)$ are int-soft PI-ideals over $U$, then so is their $\wedge$-product.

Proof. Note that $X \times Y$ is a BCK-algebra. If $(\alpha, X)$ and $(\alpha, Y)$ are int-soft PI-ideals over $U$, then $(\alpha, X)$ and $(\alpha, Y)$ are intsoft ideals over $U$ by Theorem 9 . For any $(x, y) \in X \times Y$, we have

$$
\alpha_{X \wedge Y}(0,0)=\alpha(0) \cap \alpha(0) \supseteq \alpha(x) \cap \alpha(y)=\alpha_{X \wedge Y}(x, y)
$$

Let $\left(x_{1}, y_{1}\right),\left(x_{2}, y_{2}\right) \in X \times Y$. Then

$$
\begin{aligned}
& \alpha_{X \wedge Y}\left(x_{1}, y_{1}\right) \\
& \quad=\alpha\left(x_{1}\right) \cap \alpha\left(y_{1}\right) \\
& \quad \supseteq\left(\alpha\left(x_{1} * x_{2}\right) \cap \alpha\left(x_{2}\right)\right) \cap\left(\alpha\left(y_{1} * y_{2}\right) \cap \alpha\left(y_{2}\right)\right) \\
& \quad=\left(\alpha\left(x_{1} * x_{2}\right) \cap \alpha\left(y_{1} * y_{2}\right)\right) \cap\left(\alpha\left(x_{2}\right) \cap \alpha\left(y_{2}\right)\right) \\
& \quad=\alpha_{X \wedge Y}\left(x_{1} * x_{2}, y_{1} * y_{2}\right) \cap \alpha_{X \wedge Y}\left(x_{2}, y_{2}\right) \\
& \quad=\alpha_{X \wedge Y}\left(\left(x_{1}, y_{1}\right) *\left(x_{2}, y_{2}\right)\right) \cap \alpha_{X \wedge Y}\left(y_{1}, y_{2}\right) .
\end{aligned}
$$

Therefore $\left(\alpha_{X \wedge Y}, X \times Y\right)$ is an int-soft ideal over $U$. Also, we have

$$
\begin{aligned}
& \alpha_{X \wedge Y}\left(\left(x_{1}, y_{1}\right) *\left(x_{2}, y_{2}\right)\right) \\
& \quad=\alpha_{X \wedge Y}\left(x_{1} * x_{2}, y_{1} * y_{2}\right) \\
& \quad=\alpha\left(x_{1} * x_{2}\right) \cap \alpha\left(y_{1} * y_{2}\right) \\
& \quad \supseteq \alpha\left(\left(x_{1} * x_{2}\right) * x_{2}\right) \cap \alpha\left(\left(y_{1} * y_{2}\right) * y_{2}\right) \\
& \quad=\alpha_{X \wedge Y}\left(\left(x_{1} * x_{2}\right) * x_{2},\left(y_{1} * y_{2}\right) * y_{2}\right) \\
& \quad=\alpha_{X \wedge Y}\left(\left(\left(x_{1}, y_{1}\right) *\left(x_{2}, y_{2}\right)\right) *\left(x_{2}, y_{2}\right)\right) .
\end{aligned}
$$

It follows from Theorem 12 that $\left(\alpha_{X \wedge Y}, X \times Y\right)$ is an int-soft PI-ideal over $U$.

The following example shows that the $\vee$-product of intsoft PI-ideals is not an int-soft PI-ideal.

Example 27. Consider two BCK-algebras $X=\{0,1,2\}$ and $Y=\{0, a, b\}$ with the following Cayley tables:

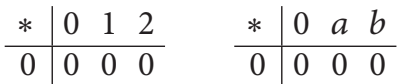

$$
\begin{aligned}
& \begin{array}{lllllllll}
1 & 1 & 0 & 1 & & a & a & 0 & 0
\end{array} \\
& 2 \mid \begin{array}{llll|lll}
2 & 2 & 0 & b & b & b & 0
\end{array}
\end{aligned}
$$

Then $X \times Y=\{(0,0),(0, a),(0, b),(1,0),(1, a),(1, b),(2,0)$, $(2, a),(2, b)\}$ is a BCK-algebra with the following Cayley table:

\begin{tabular}{c|ccccccccc}
$*$ & $(0,0)$ & $(0, a)$ & $(0, b)$ & $(1,0)$ & $(1, a)$ & $(1, b)$ & $(2,0)$ & $(2, a)$ & $(2, b)$ \\
\hline$(0,0)$ & $(0,0)$ & $(0,0)$ & $(0,0)$ & $(0,0)$ & $(0,0)$ & $(0,0)$ & $(0,0)$ & $(0,0)$ & $(0,0)$ \\
$(0, a)$ & $(0, a)$ & $(0,0)$ & $(0,0)$ & $(0, a)$ & $(0,0)$ & $(0,0)$ & $(0, a)$ & $(0,0)$ & $(0,0)$ \\
$(0, b)$ & $(0, b)$ & $(0, b)$ & $(0,0)$ & $(0, b)$ & $(0, b)$ & $(0,0)$ & $(0, b)$ & $(0, b)$ & $(0,0)$ \\
$(1,0)$ & $(1,0)$ & $(1,0)$ & $(1,0)$ & $(0,0)$ & $(0,0)$ & $(0,0)$ & $(1,0)$ & $(1,0)$ & $(1,0)$ \\
$(1, a)$ & $(1, a)$ & $(1,0)$ & $(1,0)$ & $(0, a)$ & $(0,0)$ & $(0,0)$ & $(1, a)$ & $(1,0)$ & $(1,0)$ \\
$(1, b)$ & $(1, b)$ & $(1, b)$ & $(1,0)$ & $(0, b)$ & $(0, b)$ & $(0,0)$ & $(1, b)$ & $(1, b)$ & $(1,0)$ \\
$(2,0)$ & $(2,0)$ & $(2,0)$ & $(2,0)$ & $(2,0)$ & $(2,0)$ & $(2,0)$ & $(0,0)$ & $(0,0)$ & $(0,0)$ \\
$(2, a)$ & $(2, a)$ & $(2,0)$ & $(2,0)$ & $(2, a)$ & $(2,0)$ & $(2,0)$ & $(0, a)$ & $(0,0)$ & $(0,0)$ \\
$(2, b)$ & $(2, b)$ & $(2, b)$ & $(2,0)$ & $(2, b)$ & $(2, b)$ & $(2,0)$ & $(0, b)$ & $(0, b)$ & $(0,0)$
\end{tabular}

Let $U$ be the set of alphabets. Let $(\alpha, X)$ and $(\alpha, Y)$ be soft sets over $U$ given by

$$
\begin{aligned}
\alpha: X \longrightarrow \mathscr{P}(U), & x \longmapsto \begin{cases}\{p, q, r, u, v\} & \text { if } x \in\{0,1\}, \\
\{p, q\} & \text { if } x=2,\end{cases} \\
\alpha: Y \longrightarrow \mathscr{P}(U), & x \longmapsto \begin{cases}\{p, q, r, u, v\} & \text { if } x=0, \\
\{q, r, u\} & \text { if } x=a, \\
\{r, u\} & \text { if } x=b,\end{cases}
\end{aligned}
$$

respectively. Then $(\alpha, X)$ and $(\alpha, Y)$ are int-soft PI-ideals over $U$. But the $\vee$-product $\left(\alpha_{X \vee Y}, X \times Y\right)$ is not an int-soft PI-ideal over $U$. Note that

$$
\begin{aligned}
\alpha_{X \vee Y}(2, b) & =\{p, q, r, u\} \nsupseteq\{p, q, r, u, v\} \\
& =\alpha_{X \vee Y}((2, b) *(1, b)) \cap \alpha_{X \vee Y}(1, b),
\end{aligned}
$$

and so $\left(\alpha_{X \vee Y}, X \times Y\right)$ is not an int-soft ideal over $U$. Thus $\left(\alpha_{X \vee Y}, X \times Y\right)$ is not an int-soft PI-ideal over $U$.

Let $(\alpha, X)$ and $(\beta, X)$ be soft sets over $U$. The soft intersection of $(\alpha, X)$ and $(\beta, X)$ is defined to be a soft set $(\alpha \widetilde{\cap} \beta, X)$ over $U$ which is defined by

$$
\alpha \widetilde{\cap} \beta: X \longrightarrow \mathscr{P}(U), \quad x \longmapsto \alpha(x) \cap \beta(x)
$$

The soft union of $(\alpha, X)$ and $(\beta, X)$ is defined to be a soft set $(\alpha \widetilde{U} \beta, X)$ over $U$ which is defined by

$$
\alpha \tilde{\cup} \beta: X \longrightarrow \mathscr{P}(U), \quad x \longmapsto \alpha(x) \cup \beta(x)
$$

Theorem 28. If $(\alpha, X)$ and $(\beta, X)$ are int-soft PI-ideals over $U$, then so is their soft intersection $(\alpha \widetilde{\cap} \beta, X)$.

Proof. Assume that $(\alpha, X)$ and $(\beta, X)$ are int-soft PI-ideals over $U$. For any $x, y$, and $z \in X$, we have

$$
\begin{gathered}
(\alpha \tilde{\cap} \beta)(0)=\alpha(0) \cap \beta(0) \supseteq \alpha(x) \cap \beta(x)=(\alpha \widetilde{\cap} \beta)(x), \\
(\alpha \widetilde{\cap} \beta)(x * z)=\alpha(x * z) \cap \beta(x * z)
\end{gathered}
$$




$$
\begin{aligned}
& \supseteq(\alpha((x * y) * z) \cap \alpha(y * z)) \\
& \cap(\beta((x * y) * z) \cap \beta(y * z)) \\
& =(\alpha((x * y) * z) \cap \beta((x * y) * z)) \\
& \cap(\alpha(y * z) \cap \beta(y * z)) \\
& =(\alpha \widetilde{\cap} \beta)((x * y) * z) \cap(\alpha \tilde{\cap} \beta)(y * z) .
\end{aligned}
$$

Therefore $(\alpha \widetilde{\cap} \beta, X)$ is an int-soft PI-ideal over $U$.

The following example shows that the soft union of intsoft PI-ideals is not an int-soft PI-ideal over $U$.

Example 29. Let $X=\{0,1,2,3,4\}$ be a BCK-algebra with the following Cayley table:

\begin{tabular}{l|lllll}
$*$ & 0 & 1 & 2 & 3 & 4 \\
\hline 0 & 0 & 0 & 0 & 0 & 0 \\
1 & 1 & 0 & 1 & 0 & 0 \\
2 & 2 & 2 & 0 & 0 & 0 \\
3 & 3 & 2 & 1 & 0 & 0 \\
4 & 4 & 4 & 4 & 4 & 0
\end{tabular}

Let $U$ be the set of alphabets. Let $(\alpha, X)$ and $(\beta, X)$ be soft sets over $U$ given by

$$
\begin{aligned}
& \alpha: X \longrightarrow \mathscr{P}(U), x \longmapsto \begin{cases}\{a, c, d\} & \text { if } x=0, \\
\{a, c\} & \text { if } x=1, \\
\{a\} & \text { if } x \in\{2,3,4\},\end{cases} \\
& \beta: X \longrightarrow \mathscr{P}(U), \quad x \longmapsto \begin{cases}\{b, c, d\} & \text { if } x=0, \\
\{b, c\} & \text { if } x=2, \\
\{b\} & \text { if } x \in\{1,3,4\},\end{cases}
\end{aligned}
$$

respectively. Then $(\alpha, X)$ and $(\beta, X)$ are int-soft PI-ideals over $U$, and

$$
\alpha \tilde{\cup} \beta: X \longrightarrow \mathscr{P}(U), \quad x \longmapsto \begin{cases}\{a, b, c, d\} & \text { if } x=0, \\ \{a, b, c\} & \text { if } x \in\{1,2\}, \\ \{a, b\} & \text { if } x \in\{3,4\} .\end{cases}
$$

Note that

$$
(\alpha \widetilde{\cup} \beta)(3)=\{a, b\} \nsupseteq\{a, b, c\}=(\alpha \widetilde{\cup} \beta)(3 * 1) \cap(\alpha \widetilde{\cup} \beta)(1) .
$$

Hence $(\alpha \widetilde{U} \beta, X)$ is not an int-soft ideal over $U$, and therefore $(\alpha \tilde{U} \beta, X)$ is not an int-soft PI-ideal over $U$.

\section{Conclusion}

With the aim of providing a soft algebraic tool in considering many problems that contain uncertainties, we have introduced the notion of int-soft positive implicative ideals and investigated related properties. We have considered relations between an int-soft ideal and an int-soft positive implicative ideal and established characterizations of an int-soft positive implicative ideal. We have constructed extension property for an int-soft positive implicative ideal. We have dealt with the $\wedge$-product and $\vee$-product of int-soft positive implicative ideals and discussed the soft intersection (resp., union) of intsoft positive implicative ideals. Our future research will be focused on studying the application of this structure to other algebraic structures.

\section{Acknowledgments}

The authors wish to thank the anonymous reviewer(s) for their valuable suggestions. The second author, Y. B. Jun, is an Executive Research Worker of Educational Research Institute Teachers College in Gyeongsang National University.

\section{References}

[1] D. Molodtsov, "Soft set theory-first results," Computers \& Mathematics with Applications, vol. 37, no. 4-5, pp. 19-31, 1999.

[2] D. A. Molodtsov, "The description of a dependence with the help of soft sets," Journal of Computer and Systems Sciences International, vol. 40, no. 6, pp. 977-984, 2001.

[3] D. A. Molodtsov, The Theory of Soft Sets, URSS, Moscow, Russia, 2004.

[4] D. A. Molodtsov, V. Y. Leonov, and D. V. Kovkov, "Soft sets technique and its application," Nechetkie Sistemy i Myagkie Vychisleniya, vol. 1, no. 1, pp. 8-39, 2006.

[5] U. Acar, F. Koyuncu, and B. Tanay, "Soft sets and soft rings," Computers \& Mathematics with Applications, vol. 59, no. 11, pp. 3458-3463, 2010.

[6] H. Aktaş and N. Çağman, "Soft sets and soft groups," Information Sciences, vol. 177, no. 13, pp. 2726-2735, 2007.

[7] A. O. Atagün and A. Sezgin, "Soft substructures of rings, fields and modules," Computers \& Mathematics with Applications, vol. 61, no. 3, pp. 592-601, 2011.

[8] F. Feng, Y. B. Jun, and X. Zhao, "Soft semirings," Computers \& Mathematics with Applications, vol. 56, no. 10, pp. 2621-2628, 2008.

[9] Y. B. Jun, "Soft BCK/BCI-algebras," Computers \& Mathematics with Applications, vol. 56, no. 5, pp. 1408-1413, 2008.

[10] Y. B. Jun, H. S. Kim, and J. Neggers, "Pseudo $d$-algebras," Information Sciences, vol. 179, no. 11, pp. 1751-1759, 2009.

[11] Y. B. Jun, K. J. Lee, and A. Khan, "Soft ordered semigroups," Mathematical Logic Quarterly, vol. 56, no. 1, pp. 42-50, 2010.

[12] Y. B. Jun, K. J. Lee, and C. H. Park, "Soft set theory applied to ideals in $d$-algebras," Computers \& Mathematics with Applications, vol. 57, no. 3, pp. 367-378, 2009.

[13] Y. B. Jun, K. J. Lee, and J. Zhan, "Soft $p$-ideals of soft BCIalgebras," Computers \& Mathematics with Applications, vol. 58, no. 10, pp. 2060-2068, 2009.

[14] Y. B. Jun and C. H. Park, "Applications of soft sets in ideal theory of BCK/BCI-algebras," Information Sciences, vol. 178, no. 11, pp. 2466-2475, 2008.

[15] C. H. Park, Y. B. Jun, and M. A. Öztürk, "Soft WS-algebras," Korean Mathematical Society, vol. 23, no. 3, pp. 313-324, 2008. 
[16] J. Zhan and Y. B. Jun, "Soft BL-algebras based on fuzzy sets," Computers \& Mathematics with Applications, vol. 59, no. 6, pp. 2037-2046, 2010.

[17] Y. B. Jun, M. S. Kang, and K. J. Lee, "Intersectional soft sets and applications to BCK/BCI-algebras," Journal of the Korean Mathematical Society, vol. 28, no. 1, pp. 11-24, 2013.

[18] Y. B. Jun, K. J. Lee, and E. H. Roh, "Intersectional soft BCK/BCIideals," Annals of Fuzzy Mathematics and Informatics, vol. 4, no. 1, pp. 1-7, 2012.

[19] J. Meng and Y. B. Jun, BCK-Algebras, Kyung Moon Sa, Seoul, South Korea, 1994.

[20] Y. Huang, BCI-Algebra, Science Press, Beijing, China, 2006.

[21] N. Çağman and S. Enginoğlu, "Soft set theory and uni-int decision making," European Journal of Operational Research, vol. 207, no. 2, pp. 848-855, 2010.

[22] P. K. Maji, R. Biswas, and A. R. Roy, "Soft set theory," Computers \& Mathematics with Applications, vol. 45, no. 4-5, pp. 555-562, 2003.

[23] P. K. Maji, A. R. Roy, and R. Biswas, "An application of soft sets in a decision making problem," Computers \& Mathematics with Applications, vol. 44, no. 8-9, pp. 1077-1083, 2002.

[24] Y. B. Jun, "Union soft sets with applications in BCK/BCI-algebras," Bulletin of the Korean Mathematical Society. In press. 


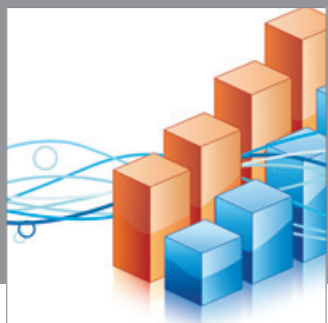

Advances in

Operations Research

mansans

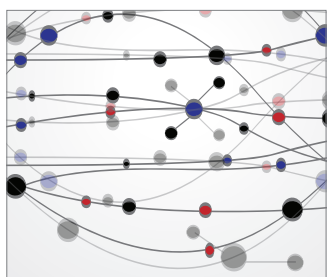

The Scientific World Journal
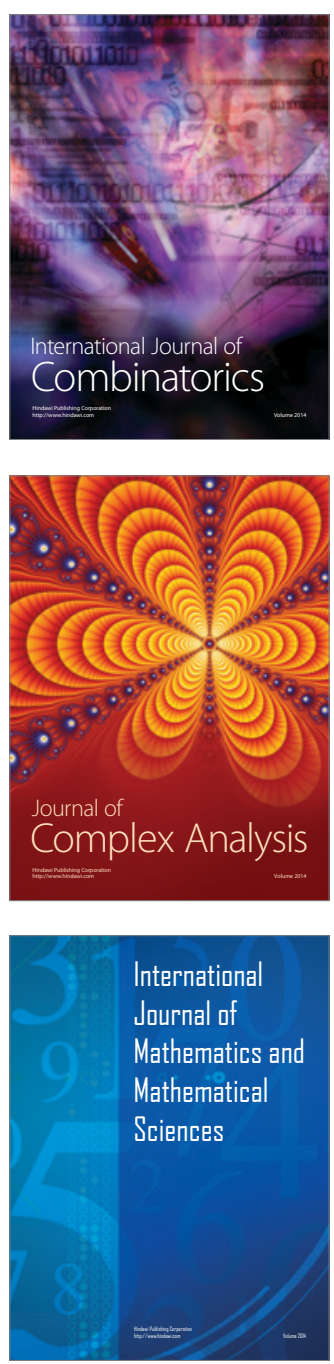
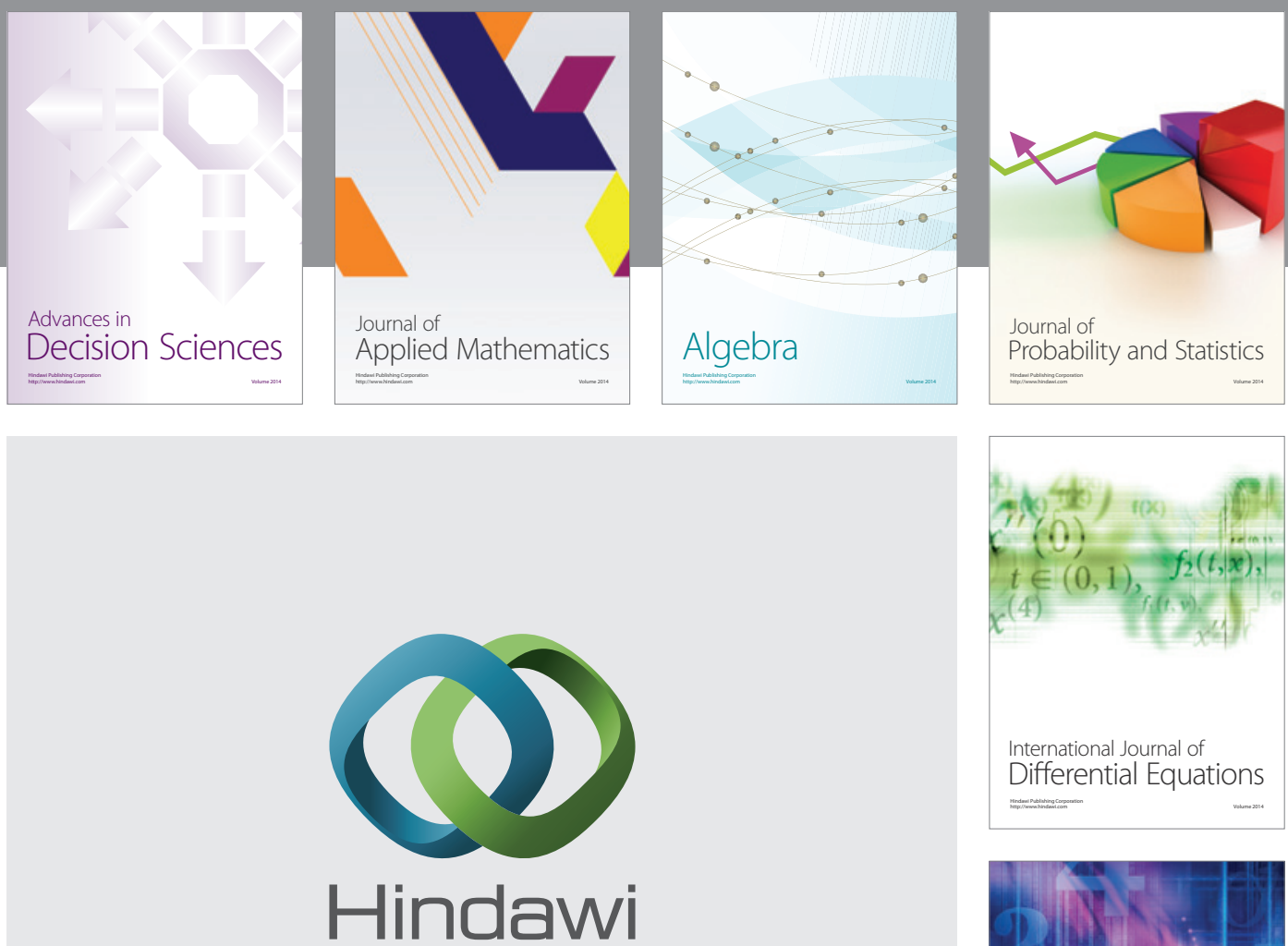

Submit your manuscripts at http://www.hindawi.com
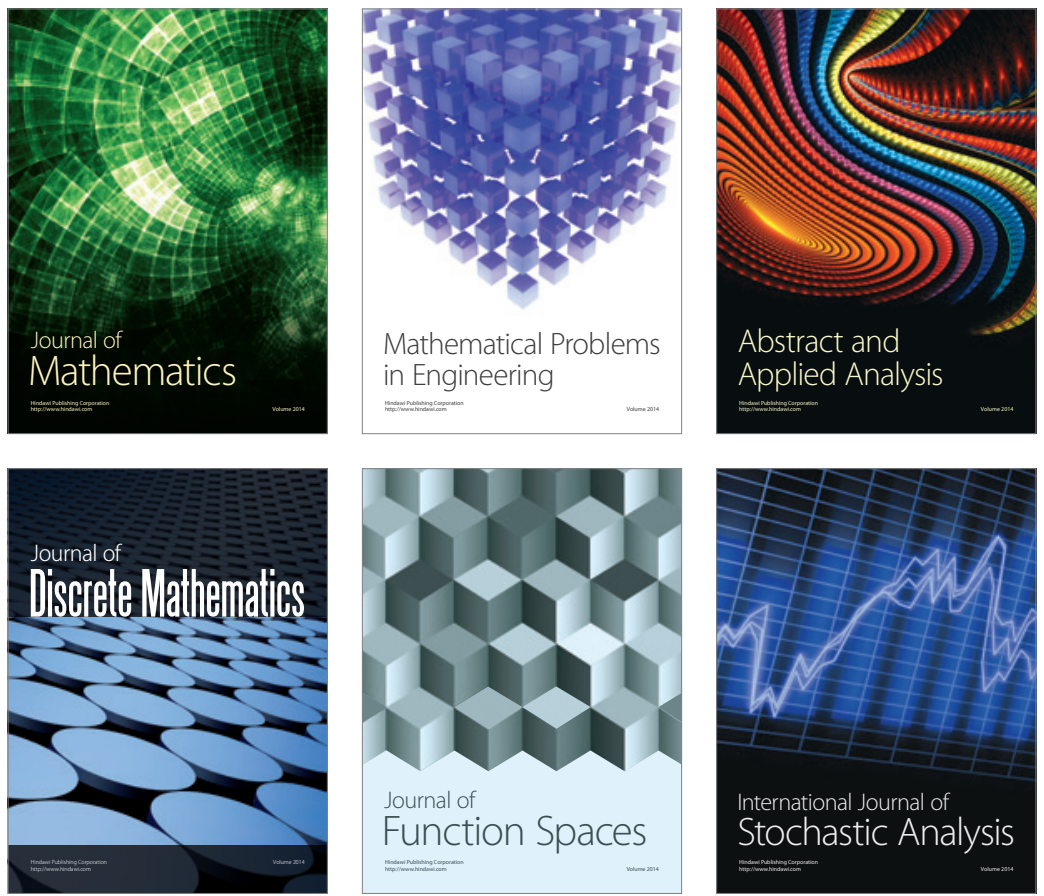

Journal of

Function Spaces

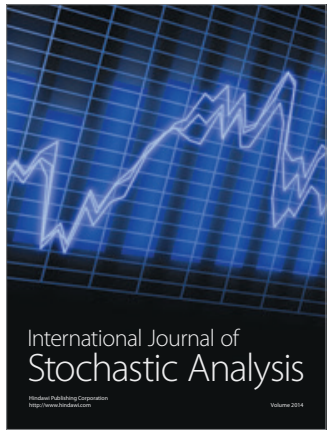

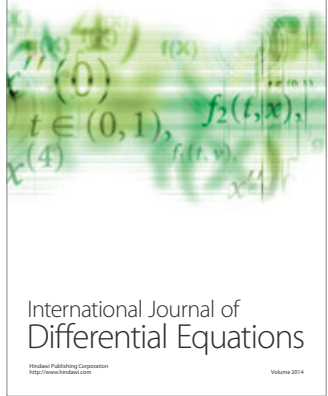
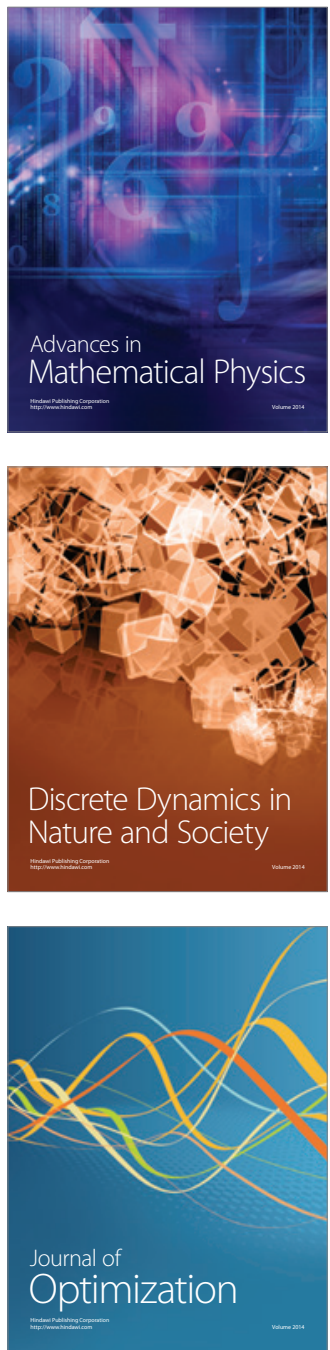